\title{
Directional Indicator on Neural Oscillations as a Measure of Synaptic Plasticity in Chronic Unpredictable Stress Rats
}

\author{
Mei Zhang ${ }^{a}$ Chenguang Zheng $^{\mathrm{a}} \quad$ Meina Quan $^{\mathrm{b}} \quad$ Lei An $^{\mathrm{a}} \quad$ Zhuo Yang $^{\mathrm{b}}$ \\ Tao Zhang ${ }^{\text {a }}$ \\ ${ }^{a}$ College of Life Sciences and Key Lab of Bioactive Materials, Ministry of Education, ${ }^{b}$ College of Medicine Science, \\ Nankai University, Tianjin, PR China
}

\section{Key Words}

Synaptic plasticity - Local field potential - IM algorithm •

Directional index $\cdot$ Neural oscillations

\begin{abstract}
To examine whether the directionality index of neural information flow (NIF) over specific oscillatory bands is useful in measuring synaptic plasticity, we employed the IM approach to determine the direction of NIF between the cortex and thalamus in normal and stressed animals. The experiment was performed by inducing long-term potentiation (LTP) of the thalamocortical pathway after recording local field potential (LFP). Additionally, comparison of IM measurement between broad- and narrowbands was performed, while a numerical study was also carried out for assessing the number of data points. The results show that the instantaneous phases extracted from narrowband vary monotonically, while these phases are jagged in broadband. Our data show that there is a predominant driving effect (coupling directional index $d>0$ ) from the thalamus to the frontal cortex in normal animals; however, the value of $d$ is significantly reduced in the chronic stressed group in both the delta and theta bands. Furthermore, the field LTP data show that
\end{abstract}

chronic stress decreases medial prefrontal cortex synaptic plasticity, which is certainly in line with the LFP findings. Together, these data suggest that using an IM algorithm, the directionality index of NIF in specific oscillatory frequency bands will probably be used as a measure of synaptic plasticity.

Copyright $\odot 2011$ S. Karger AG, Basel

\section{Introduction}

It is well known that the prefrontal cortex (PFC) is likely an important brain region in some memory processes, as it is involved in cognition, learning and memory [1]. Meanwhile, the thalamus region plays a central role in the thalamo-cortico-thalamic circuits, as it is part of the primary pathway by which information from the outside world is transmitted to the neocortex [2,3]. Recently, thalamocortical (TC) projection has been given extensive attention [1]. It is reported that the cognitive impairment

M. Zhang and C. Zheng contributed equally to this work. 
in the early stages of schizophrenia are largely caused by losses in the TC pathway [4]. Furthermore, growing recognition shows that dysfunction of the PFC and the circuitry associated with it may partly underlie the impairment of cognitive function and executive processes, including working memory and decision-making from neuropsychiatric disorders [5].

The oscillatory phenomena in the brain electrical activity and their synchronization [6] is related to cognitive processes [7], with properties changing under cognitive disorders such as schizophrenia [8], Alzheimer's disease and attention-deficit hyperactivity disorder [9]. As is well known from animal studies, neural oscillations in the delta and theta bands are the dominant rhythms in the thalamocortex of mammals, and theta oscillations reflect cognitive and memory performance [10]. Thus, particular influences will be shown on delta and theta oscillations.

Synaptic plasticity is widely believed to provide the cellular basis for most models of learning, memory and development in neural circuits [11]. Based on the mechanisms of activity-dependent alterations of excitatory synaptic transmission, synaptic plasticity points to the important roles in cognitive, learning and memory functions, with a classical functional index, long-term potentiation (LTP) $[3,12]$. It is an enduring form of synaptic plasticity in the mammalian brain [13]. Nowadays, more and more attention is being paid to LTP of the TC pathway because of its tight contact with cognitive function, which can change in depression [14-16]. It has been reported that cognitive impairment in the early stages of schizophrenia is probably attributed to a loss of connectivity between the PFC and midline-anterior thalamus [4]. A recent study from our lab demonstrated that synaptic plasticity of the laterodorsal thalamic nucleus (LD) to the PFC pathway was impaired in an animal model of psychiatric diseases [14].

With increasing synchronization and related phenomena observed in many physiological systems, many scientific efforts have focused on the importance of identifying causal (driver-response) relationships between concerned (sub)systems [15]. Recently, some coupling direction algorithms have been proposed for the analysis of neural information flow (NIF), and the knowledge of information-theoretic measures is essential [16]. An approach measuring a directionality index (abbreviated to IM in this paper) was developed [15, 17]. It was proposed, based on conditional mutual information, and applied to the instantaneous phases of weakly coupled oscillators $[15,17]$. This approach has been successfully applied not only to model systems $[15,18,19]$, but also to EEG data from epileptic patients [20] and rats in different levels of anesthesia [21], as well as to the amplitude and frequency of cardiac oscillations [22].

A number of in vitro and in vivo studies have reported that induction of LTP is optimal when the time interval between stimuli is approximately $200 \mathrm{~ms}$ [23]. It implies that theta oscillations may be involved in synaptic plasticity. Studies show that there is a relationship between neural oscillatory bands and behavioral states [7, 24]. However, few studies have directly examined the relationship between synaptic plasticity and neural activity of local field potential (LFP) in specified frequency bands. An issue has been raised as to whether the directionality index of NIF over oscillatory bands could be used as a sign of the measurement of synaptic plasticity. In the present study, we introduced IM to measure the directionality of information flow between the medial prefrontal cortex (mPFC) and thalamus, and tried to address the issue as to whether the directionality on specific oscillatory bands could be used to indicate the alternation of synaptic plasticity in the chronic unpredictable stress (CUS) animal model. This was done by inducing LTP of the TC pathway after recording LFP at the same two locations in Wistar rats of both CUS and control groups. The aims of the study were to observe the changes of information flow transfer in depression in the CUS model, and analyze whether the direction index of NIF is able to reflect the alterations of synaptic plasticity, combining the LTP results. Furthermore, the comparison of IM measurement between broad- and narrowbands was performed. In order to assess the number of data points required for a reliable estimation of directional index by IM algorithm, a numerical study was also carried out.

\section{Methods}

Animals and the CUS Procedure

Experiments were carried out on 13 male Wistar rats (180$220 \mathrm{~g}$ body weight), with food and water freely available during all stages except when establishing the model and sucrose consumption phases. After 2 days habituation to the environment, rats were divided into two groups at random: control $(n=6)$ and stressed $(n=7)$ rats. All efforts were made to minimize the number of rats used and their suffering. The experiments were performed in accordance with the guidelines of the Beijing Laboratory Animal Center, and authorized by the Ethical Commission at Nankai University.

The CUS procedure was performed for 21 days, referring to the modification method $[25,26]$. Briefly, the weekly stress regime included seven different types of stressors, which were applied in seemingly random order and at varying times as follows: water 
deprivation $(24 \mathrm{~h})$, cage tilt $\left(45^{\circ}, 7 \mathrm{~h}\right)$, electric foot-shock $(1 \mathrm{~mA}$, $10 \mathrm{~s} /$ shock) 10 times with 10 -second intervals, food deprivation (24 h), reversed light/dark cycle $(24 \mathrm{~h})$, tail pinch $(1 \mathrm{~min})$ and ice water swimming $\left(4^{\circ} \mathrm{C}, 5 \mathrm{~min}\right)$. Meanwhile, the control group animals were left undisturbed during the establishment of the model.

Body weights were measured twice a week with intervals of 3-4 days. Before CUS, rats were habituated to drinking $1 \%$ sucrose solution for 2 days by replacing normal water. Two days before CUS, all rats were deprived of food and water for $23 \mathrm{~h}$, starting at 10 a.m. And then, the next day at 9 a.m., the rats were provided the $1 \%$ sucrose solution for $1 \mathrm{~h}$. The amount each rat drank was monitored. Sucrose intake = beginning bottle weight - ending bottle weight. Sucrose consumption $=100 \% \times$ sucrose intake/body weight. This procedure was repeated on the last 2 days of each week during the CUS period.

\section{Electrophysiological Experiment}

The signals of LFP were collected concurrently from the regions of thalamus and the frontal cortex, in which stainless steel electrodes were implanted during stereotactic surgery under $30 \%$ urethane anesthesia ( $4 \mathrm{ml} / \mathrm{kg}$, i.p.; Sigma-Aldrich, St. Louis, Mo., USA). One electrode was implanted into the prelimbic area of the mPFC [AP 3.0-3.3; L 0.7-1.0; H 2.8-3.4], and the other concentric bipolar depth electrode was implanted into the laterodorsal thalamic nucleus, dorsomedial part (LDDM) [AP -2.3 to 2.8; L 1.4$2.0 ; \mathrm{H} 4.2-4.7]$. Two additional electrodes, ground and reference, were placed symmetrically over the two hemispheres of the cerebellum. The coordinates were given in millimeters relative to the bregma so as to be in conformity with the rat brain atlas [27]. The LFP recording of the two brain areas were done simultaneously, and lasted almost $5 \mathrm{~min}$ for each rat. The signals were fed into a multichannel differential amplifier and simultaneously recorded with a sampling rate of $200 \mathrm{~Hz}$.

It is well known that the Hilbert transform has a limitation that it is only suitable for the analysis of a narrow frequency-band signal $[20,28]$. In the present study, in order to obtain higher resolution, the original signals were filtered into $1-4 \mathrm{~Hz}$ (delta band) and $4-8 \mathrm{~Hz}$ (theta band), while the bandwidth of the band-pass filter was set at $1 \mathrm{~Hz}$. For comparing data obtained from directional index measurement between broad- and narrowbands, the original signals were also filtered into a broadband $(1-10 \mathrm{~Hz})$. A band-pass filter, namely finite impulse response was employed.

After the LFP recording, LTP was induced at the same two locations, with LDDM being the stimulating area and $\mathrm{mPFC}$ being the recording area. The test stimuli were delivered to the LDDM region every $30 \mathrm{~s}$ at an intensity that evoked a field excitatory postsynaptic potential (fEPSP) of $70 \%$ of its maximum (range $0.2-0.5$ $\mathrm{mA}$ ). After a 30-min baseline, high-frequency stimulation to induce LTP consisted of two series of 10 trains $(250 \mathrm{~Hz}, 200 \mathrm{~ms})$ at $0.1 \mathrm{~Hz}$, delivered at test intensity [14]. The fEPSP signals were sampled at $20 \mathrm{kHz}$ and stored as the averages of four. Its slope was measured as the average slope from 20 to $80 \%$ of the first positive deflection of the potential.

\section{Neural Information Flow Analysis}

A directionality index based on conditional mutual information is proposed for application to the instantaneous phases of weakly coupled oscillators [15]. Its abilities to reveal and quantify asymmetry in bidirectional coupling were proved using numeri- cal examples of quasiperiodic, chaotic and noisy oscillators, as well as real EEG data [15]. Generally, the mutual information $I(X ; Y)$ of two random variables $X$ and $Y$ is given by

$$
I(X ; Y)=H(X)+H(Y)-H(X, Y) .
$$

Given the variable $Z$, the conditional mutual information is defined as

$$
I(X ; Y \mid Z)=H(X \mid Z)+H(Y \mid Z)-H(X, Y \mid Z) .
$$

Supposing two processes $\left\{X_{1}\right\}$ and $\left\{X_{2}\right\}$, their instantaneous phases $\Phi_{1}(t)$ and $\Phi_{2}(t)[29,30]$ can be estimated by application of the discrete Hilbert transform [31], which obtains the phase approximately from the original broadband signals in a frequency adaptive manner. Next, we can estimate the 'net' information about the $\tau$-future of the process $\left\{X_{1}\right\}$ contained in process $\left\{X_{2}\right\}$ (or the $\tau$-future of the process $\left\{X_{2}\right\}$ contained in process $\left\{X_{1}\right\}$ ) using $I\left(\Phi_{2} ; \Phi_{1 \tau} \mid \Phi_{1}\right)$ [or $\left.I\left(\Phi_{1} ; \Phi_{2 \tau} \mid \Phi_{2}\right)\right]$ to infer the coupling directionality [15].

To establish possible causality relations, we consider phase increments

$$
\Delta_{\tau} \Phi_{1,2}=\Phi_{1,2}(t+\tau)-\Phi_{1,2}(t)
$$

Then, the conditional mutual information is $I\left(\Phi_{1,2}(t)\right.$; $\left.\Delta_{\tau} \Phi_{2,1} \mid \Phi_{2,1}(t)\right)$. Finally, the directionality index is calculated by

$$
D(1,2)=\frac{i(1 \rightarrow 2)-i(2 \rightarrow 1)}{i(1 \rightarrow 2)+i(2 \rightarrow 1)}
$$

where the measure $i(1 \rightarrow 2)$ and $i(2 \rightarrow 1)$ are defined using the conditional mutual information $I\left(\Phi_{1}(t) ; \Delta_{\tau} \Phi_{2} \mid \Phi_{2}(t)\right)$ and $I\left(\Phi_{2}(t)\right.$; $\left.\Delta_{\tau} \Phi_{1} \mid \Phi_{1}(t)\right) . D(1,2)$ should be positive if system 1 drives system 2 , and negative for the opposite case. $D(1,2)=0$ means that the interactions between the two systems are symmetrical $[21,22]$.

\section{Data and Statistical Analysis}

All data are expressed as means \pm SEM. Two-way repeated measures ANOVA was used for sucrose consumption. Student's t test was performed on the data from the individual day/session. For the LTP test, fEPSP slopes were expressed as the percentage change from baseline. Two-way repeated measures ANOVA was applied for analysis of differences between two groups. Statistical comparisons were made using the independent samples T test, as appropriate. For the LFP data, analyses were performed using SPSS 16.0 software, and the significance level was set at 0.05 .

\section{Results}

\section{Reduction of Body Weight Gain, Sucrose Intake and Consumption in CUS}

During the 3 weeks of CUS, rats in both groups gained weight, but rats gained significantly more weight in the control group than those in the stress group $(p<0.001)$. Two-way repeated measures ANOVA confirmed the statistical difference of day $\left(F_{(6,60)}=347.548, \mathrm{p}<0.001\right)$, day 
Fig. 1. The effects of bandwidth on the instantaneous phase and directional index measurement in normal Wistar rats. a Instantaneous phase performed to be a monotonic curve almost within each period in the narrowband case. b Instantaneous phase was jagged in the broadband case. c Comparison of IM measurement between narrowband (directional index $d=0.17$ ) and broadband (directional index $d=0.03$ ). Obviously, it is only in the narrowband case in which the data suggest that cortex neurons are driven by thalamus ones. ${ }^{* *} \mathrm{p}<0.01$ comparison between narrowband and broadband.

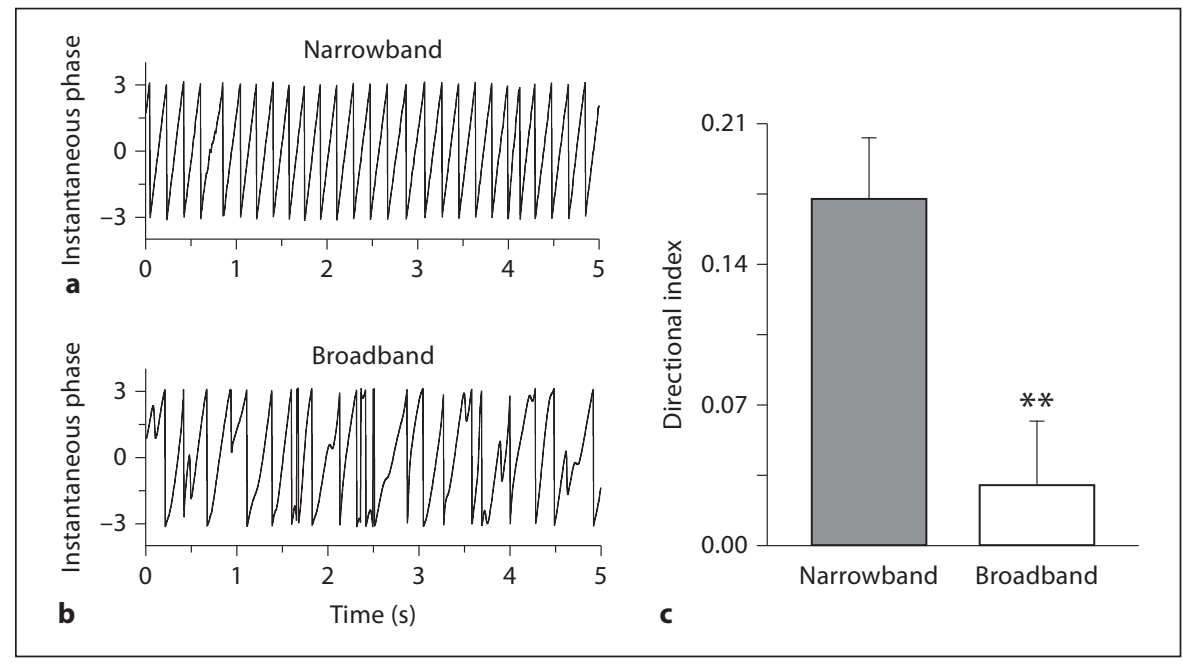

$\times$ group interaction $\left(F_{(6,60)}=147.570, \mathrm{p}<0.001\right)$, and group $\left(F_{(1,10)}=85.459, \mathrm{p}<0.001\right)$. Student's t test showed that there was significant difference between groups in all days except the 1st day $(\mathrm{p}>0.05)$. Similar results were obtained on sucrose intake and sucrose consumption (data not shown).

\section{More Reliable Measurement of Directional Index in Narrowband Phase Signals}

Comparison of directional index measurement between broad and narrow frequency bands was performed. The original signals were filtered into a $1-10-\mathrm{Hz}$ frequency band, in which broadband signals were generated. In the meantime, they were also filtered using a 1-Hz-wide band-pass filter, in which narrowband signals were obtained. Instantaneous phases were then extracted using the Hilbert transform. Figure la shows that a time series of the instantaneous phase from the narrowband varies monotonically within each period. Another time series of instantaneous phase from the broadband (fig. 1b), however, fluctuates in a jagged way, which obviously does not meet the precondition of the Hilbert transform.

A numerical test was performed to compare directional index measurement between the narrow- and broadbands. We employed a moving-window with a length of $40 \mathrm{~s}(8,000$ sample points) with an overlap of $50 \%, 8$ quantization levels $(\mathrm{q}=8)$ and time lags from 5 to 40 samples with increments of 5 . To obtain the directional index in narrowband, the LFP signals were filtered into $1-10 \mathrm{~Hz}$, using a 1-Hz-wide band-pass filter. Based on each phase's time series extracted by the Hilbert transform, the direc-

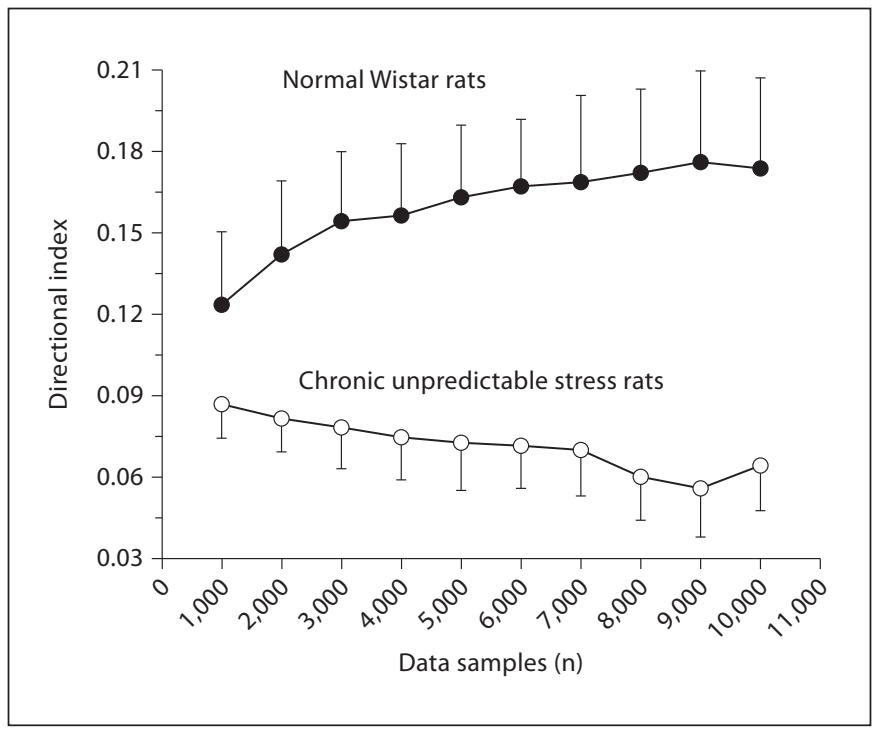

Fig. 2. Effect of record length on directional index measurement. The directional index presents as functions of record length in the range of 1,000-10,000 data points with increments of 1,000 in both the control and stressed groups.

tional index was measured, and then a total of nine indices were averaged as the directional index of narrowband signals. Figure 1c shows the group data of directional index in both narrowband and broadband states in the control group. It can be seen that the value of directional index decreased from 0.172 in narrowband to 0.030 in broadband $(\mathrm{p}<0.01)$. 
Fig. 3. The effect of chronic stress on directional index measurement of the delta and theta frequency bands. The value of $d$ in the theta band was higher than in the delta band in normal animals. a Statistical difference of the directional index $d$ in the delta bands between normal and stressed rats $(0.123 \pm 0.019$ vs. $0.009 \pm 0.032)$. b Significant difference of the directional index $d$ in the theta bands between these two groups $(0.194 \pm 0.038$ vs. $0.088 \pm$ $0.018) .{ }^{*} \mathrm{p}<0.05$ and ${ }^{* *} \mathrm{p}<0.01$ comparison between the control group and the stressed group.

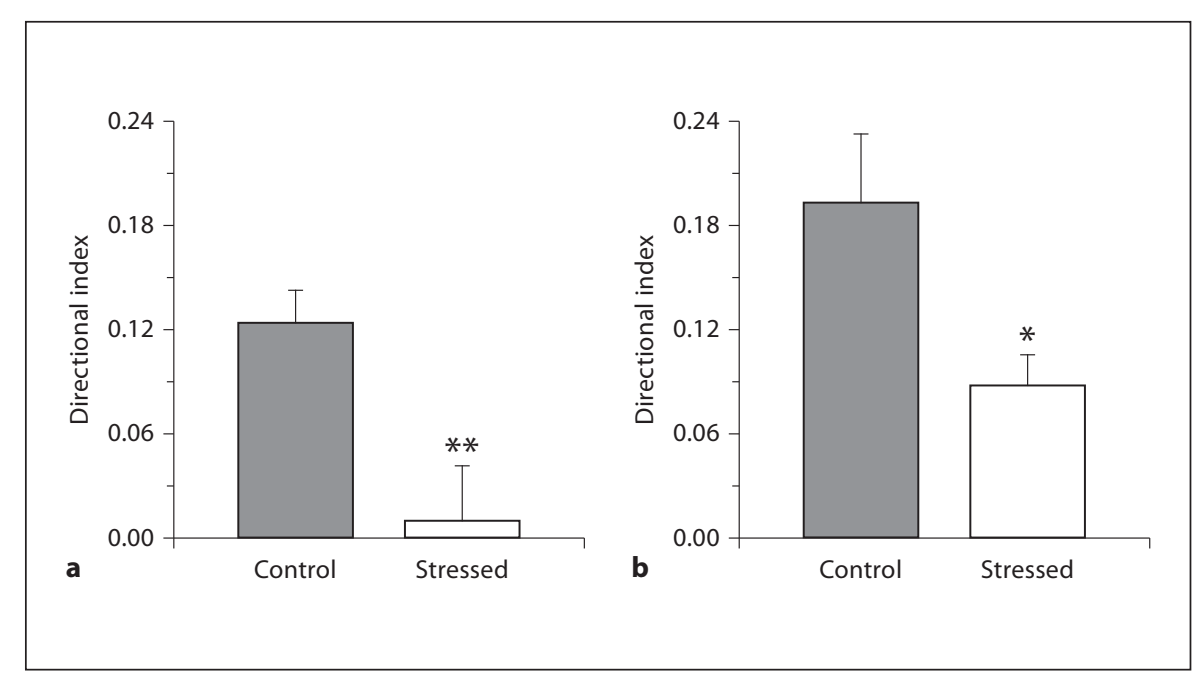

\section{Convergence of Directional Index Calculation over} 8,000 Data Points

In order to assess the number of data points required for a reliable estimation of the directional index by the IM algorithm, a numerical test was performed by increasing a moving window from a length of 1,000 to 10,000 . Figure 2 shows the values of the directional index as functions of record length in the range of 1,000-10,000 data points with increments of 1,000 in both the control and stressed groups. It can be seen that both of the curves, estimated from the time series of the instantaneous phase at narrowband, contain a small linear segment in which the slope converges to constant values as the number of data points increase (number of data points is from 8,000 to 10,000 in this plot). Such a result is consistent with a paper published in 2005 by Yokota [32], who reported that the entropy value estimated by the conventional Shannon method will not get close to the true value until the samples reach 5,000.

\section{Reduction of NIF Directional Index in CUS Rats}

The instantaneous phase within a narrowband was extracted by means of the Hilbert transform and directional index $d$ was measured by the IM approach between two time series obtained from the thalamus and cortex. However, before calculating the directionality index, the following parameters of the algorithm had to be determined: a moving window with a length of $40 \mathrm{~s}(8,000$ samples) with an overlap of $50 \%$, eight quantization levels $(\mathrm{q}=8)$ and time lags from 5 to 40 samples with increments of 5 . Figure 3 shows IM analysis of thalamus-PFC pathway in rats between the normal and stressed states in the delta and theta bands, respectively. It was found that the value of $d$ in the theta band was higher than that in the delta band in normal animals (fig. 3). Moreover, there was a statistical difference of the directional index $d$ in the delta band between normal Wistar rats and stressed rats $[0.123 \pm 0.019(\mathrm{n}=6)$ vs. $0.009 \pm 0.032(\mathrm{n}=7), \mathrm{p}<$ 0.01; fig. 3a]. A significant difference of the directional index $d$ in the theta band between these two groups can also be seen $[0.194 \pm 0.038(\mathrm{n}=6)$ vs. $0.088 \pm 0.018(\mathrm{n}=$ 7), $\mathrm{p}<0.05$; fig. 3b].

\section{Reduction of Long-Term Potential in CUS Rats}

During the LTP experiment, stimulation of LDDM evoked a basal excitatory fEPSP in the prelimbic area of the $\mathrm{mPFC}$ and high-frequency stimulation induced LTP of the stimulated synapses for at least $2 \mathrm{~h}$. Data representing the time course of fEPSP slopes normalized to the 30 -min baseline period is shown in figure $4 \mathrm{a}$. The fEPSP slopes increased immediately after the high-frequency stimulation and stabilized to a level above the baseline period. An example of fEPSP at baseline and tetanized conditions of a normal rat can be seen in the inset of figure 4a. Two-way repeated measures ANOVA showed statistical difference for group interaction $\left(F_{(1,10)}=7.326, \mathrm{p}<0.05\right)$, but no statistical difference for time $\left(F_{(5,50)}=1.189, \mathrm{p}>0.05\right)$ or time $\times$ group interaction $\left(F_{(5,50)}=1.258, \mathrm{p}>0.05\right)$. For further statistical analysis, Student's t test of the mean fEPSP slope of the 6 time points was applied in the two groups, which showed that the average fEPSP slope was statistically smaller in stressed group compared with that in the control group $\left(t_{(10)}=8.513, \mathrm{p}<0.001\right.$; fig. $\left.4 \mathrm{~b}\right)$. 


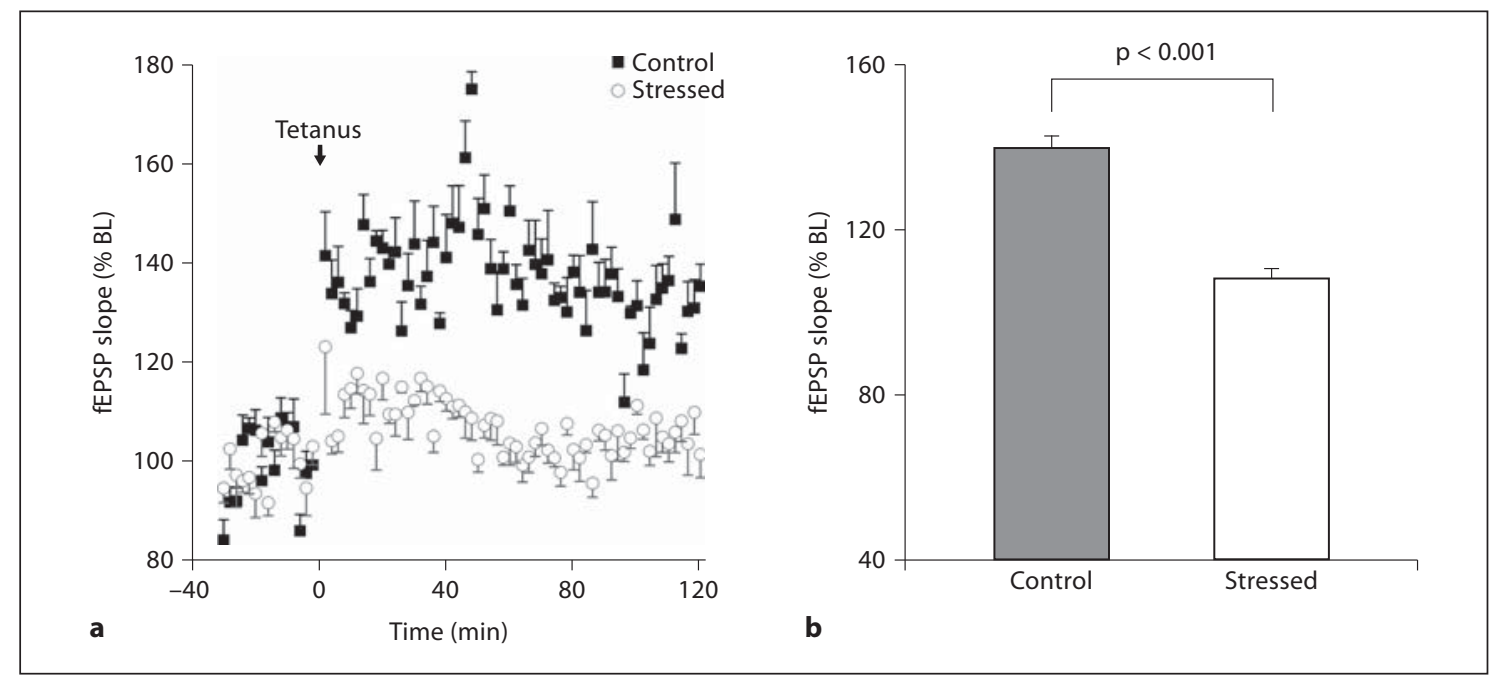

Fig. 4. The effect of chronic stress on fEPSP slopes of LTP. a Time course changes in fEPSP slopes. The fEPSP slope is plotted as a percentage change against the baseline $(30 \mathrm{~min})$ before high-frequency tetanic stimulation in the control and stressed groups. Each point represents the mean \pm SEM averaged to four consecutive evoked responses. Tetanic stimulation is indicated by an arrow. The sampling rate of fEPSP recording was $20 \mathrm{kHz}$. b Comparison of averaged fEPSP slopes of six time points (every $20 \mathrm{~min}$ ) in the two groups. The data showed that mean fEPSP slopes in the stressed group were significantly decreased.

\section{Discussion}

In this study, the CUS animal model was established to mimic clinical depression successfully via the findings of lower sucrose consumption and retarded weight gain. CUS is widely accepted as an animal model of depression [33], which reflected anhedonia or a decrease in the rewarding properties of the solution [34]. Based on the signals of the LFP collected concurrently from the regions of thalamus and the frontal cortex, the IM algorithm was applied to measuring the bidirectional (straightforward and backward) information flow on the thalamus-PFC pathway. In order to exhibit the change of synaptic plasticity in the frontal cortex in depression, we undertook electrophysiological experiments and induced LTP through the TC pathway. In addition, a numerical test was performed in order to verify the influence of the bandwidth of the neural signals on the instantaneous phase and further compare the directional index measurement between narrow- and broadbands, as well as choose a reasonable data length for the moving window.

It has been reported that active TC synapses drive the cortex weakly but synchronously [2], and the signals of LFP recorded from the two regions interact with each other by weak coupling. Taking the existing physical conclusions into account that weak coupling first has influ- ence on the phase of oscillators rather than their amplitudes $[29,30]$, we extracted the instantaneous phase sequences by which LFP signals were analyzed. In the present study, an IM algorithm was employed to calculate the direction of NIF on the thalamus-PFC pathway. It is well known that there are several approaches by which phase signals can be extracted; in this study the Hilbert transform was employed. Since LFP represents the sum of a very large number of neuronal dendritic potentials in the brain [35], original LFP has a broad frequency with abundant noises. Thus, it may not perform as an incremental curve if the phase sequence is extracted by the Hilbert transform directly without preprocessing, and thereby not meet the precondition of the Hilbert transform. The results show that the instantaneous phases extracted from narrowband vary monotonically within each period while those extracted from broadband are jagged, which are consistent with the fact that the Hilbert transform is only suitable to narrow frequency-band signals [28]. Therefore, it can be concluded that the measurement of the directional index may be reliable in the condition of using narrowband phase signals.

Conditional mutual information in the IM algorithm is based on traditional Shannon entropy [17]. Previous studies suggest that entropy value estimated by the conventional Shannon method will approximate to the true 
value when the number of samples reaches 5,000 [32]. The numerical test was performed to measure the directionality of NIF with changing the data length of the moving window in both the control and stressed groups. It showed that the result converged to a constant as the number of data points increased, which was consistent with the number of data points reported in the study [32].

There have been previous reports in the literature that cognitive function is closely related to neural oscillatory rhythms, such as delta and theta oscillations, which characterize slow-wave sleep and mainly reflect thalamocortical interactions $[10,36]$ as the dominant rhythms in the TC pathway of mammals. Theta oscillations were thought to be associated with some kinds of cognitive functions, such as learning and working memory, and especially for encoding processes [10]. Furthermore, it was reported that in bipolar 'euthymic' patients, unmedicated patients have higher delta amplitudes than healthy subjects [9]. Therefore, only the signals in the delta $(1-4 \mathrm{~Hz})$ and theta bands $(4-8 \mathrm{~Hz})$ were extracted from the original LFP in the present study.

It is reasonable to apply the IM algorithm in measuring the direction of NIF between the thalamus and PFC because active TC synapses drive the cortex weakly [2]. Our data show that there is a predominant driving effect $(d>0)$ from the thalamus to the frontal cortex in both the delta band and the theta band in the control group. Such a result is not only in accord with the findings reported in healthy objects $[37,38]$, but also consistent with that obtained by phase dynamical analysis [39]. In vivo thalamic neurons have higher spontaneous and evoked firing rates compared with cortical neurons [40,41], which may explain why the information transferred from the thalamus to the cortex in the control group. However, the coupling direction index $d$ in either the delta band or the theta band was significantly reduced in the chronic stressed group, which indicated that the pattern of NIF was influenced by depression in the CUS animal model. Moreover, it suggested that the cognitive deficits were partly caused by weakened TC information transfer. Previous studies have shown that TC connectivity was prominent in the resting state in healthy controls, but changed in schizophrenia [37]. Furthermore, it has been reported that the fiber pathways connecting the midline and anterior thalamic nuclei to the PFC had a decrease in schizophrenia in magnetic resonance imaging $[4,42]$. All these findings are consistent with our data in some way.

The alterations in synaptic plasticity of the TC pathway might be a crucial mechanism that caused the impairment of cognitive function in schizophrenic rats as definitive synaptic projections were found from the LD thalamus to the MPFC by LTP experiments in both healthy and schizophrenic groups [14]. In vivo recordings of action potentials emitted by presynaptic thalamic cells and fEPSP in postsynaptic cortical cells indicate that active TC synapses drive the cortex weakly but synchronously [2]. Moreover, large fEPSPs were found by minimal electrical stimulation of TC fiber tracts in in vitro recordings from cortical neurons in acute (fresh) brain slices [43]. In the present study, similar results were obtained from a different animal model. Our LTP results showed that fEPSP slopes in the stressed group decreased after high-frequency stimulus of the LD thalamus, which validated that chronic stress could result in reduction of synaptic plasticity in mPFC. Thus, it suggested that the cognitive deficits caused by chronic stress were partially attributed to the reduction of synaptic plasticity and weakened connection in TC pathway. Adding to the preceding report, it indicated that the modifications of synaptic plasticity related to LTP were required for the behavioral or cognitive plasticity generated by the experience [44].

Since the information flow gives a dynamic measurement and the synaptic connectivity describes the static character of the network [45], we understand that the change of NIF may be related to the alterations in synaptic plasticity of the TC pathway. Since spontaneous neural discharges in mPFC were affected by those in the LD thalamus through the TC pathway on which LTP had been successfully induced, it concluded that the direction of NIF on the TC pathway was changed in both the delta and theta oscillatory frequency bands in chronic stressed rats, which was related to the reduction of synaptic plasticity in this pathway. Moreover, it suggests that neural oscillations, especially in the delta and theta bands, have a close relation to cognitive function in a different aspect.

To better investigate the relation between NIF and LTP, the control experiments were performed to measure and compare the patterns of NIF before and after the induction of LTP. It was found that there was no significant difference of the directionality index $d$ between pre-LTP LFP and post-LTP LFP in either the delta or theta frequency bands. We understand that directionality index $d$ reflects the direction of NIF coupling between the thalamus and PFC. After high-frequency electrical stimulation, the patterns of LFP in both sites might be changed linearly; however, it is not necessary that there should be pattern alteration in a nonlinear way. The directional indicator $d$ is a nonlinear index; therefore, it is comprehensible that there is no statistical difference of $d$ between 
pre-LTP LFP and post-LTP LFP. Moreover, LFP and LTP are different kinds of electrophysiological information. LTP might show the information of a specific neural pathway, such as the TC pathway in the present study. We understand that there is relatively broader and richer information within LFP, as the interaction between the thalamus and cortex represents not only the direct pathway from the thalamus to the PFC, but also other indirect neural pathways and circuits. Nevertheless, despite the difference, the present study still suggests that LFP and LTP might have something in common, and in some level they are correlated with each other.

In summary, we raised the question as to whether the directionality index of NIF over specific frequency bands could be used as a sign of the measurement of synaptic plasticity. The results showed that the coupling direction index was significantly reduced in stressed state compared to that in normal state only in the theta band, but also in delta band. It suggested that the pattern of NIF was drastically affected in the CUS animal model on these two oscillatory frequency bands. It further suggested that the cognitive dysfunction could be in part caused by the reduction of information transfer along the TC pathway and that both theta and delta oscillations were involved with these processes. Meanwhile, the LTP experiments showed that chronic stress diminished mPFC synaptic plasticity, which was undeniably in line with the LFP findings. Taken together, these data suggested that based on an IM algorithm, the directionality index of NIF in specific oscillatory frequency bands will probably be used as a measure of synaptic plasticity. However, studying the relationship between the coupling direction index of NIF and synaptic plasticity is still at an early stage of development. It remains an open question as to whether there are other oscillatory frequency bands involved to index the changes of synaptic plasticity and how they perform during this process.

\section{Acknowledgements}

This work was supported by grants from the National Natural Science Foundation of China (30870827, 31070964).

\section{References}

-1 Izaki Y, Takita M, Nomura M, Akema T: Effects of hippocampus-induced prefrontal long-term depression on gamma-band local field potential in anesthetized rats. Neurosci Lett 2002;330:204-206.

$>2$ Bruno RM, Sakmann B: Cortex is driven by weak but synchronously active thalamocortical synapses. Science 2006;312:1622-1627.

-3 Ng WX, Lau IY, Graham S, Sim K: Neurobiological evidence for thalamic, hippocampal and related glutamatergic abnormalities in bipolar disorder: a review and synthesis. Neurosci Biobehav Rev 2009;33:336-354.

4 Lambe E, Liu R, Aghajanian G: Schizophrenia, hypocretin (orexin), and the thalamocortical activating system. Schizophr Bull 2007;33:1284-1290.

5 Dalley J, Cardinal R, Robbins T: Prefrontal executive and cognitive functions in rodents: neural and neurochemical substrates. Neurosci Biobehav Rev 2004;28:771-784.

$\checkmark 6$ Vejmelka M, Palus M: Detecting nonlinear oscillations in broadband signals. Chaos 2009; 19:015114.

7 Ward L: Synchronous neural oscillations and cognitive processes. Trends Cogn Sci 2003; 7:553-559.

$>8$ Bob P, Palus M, Susta M, Glaslova K: EEG phase synchronization in patients with paranoid schizophrenia. Neurosci Lett 2008;447: 73-77.
-9 Basar E, Güntekin B: A review of brain oscillations in cognitive disorders and the role of neurotransmitters. Brain Res 2008;1235: 172-193.

$>10$ Klimesch W: EEG alpha and theta oscillations reflect cognitive and memory performance: a review and analysis. Brain Res Rev 1999;29:169-195.

11 Abbott L, Nelson S: Synaptic plasticity: taming the beast. Nature Neurosci 2000;3:11781183.

12 Lante F, de Jesus Ferreira MC, Guiramand J, Recasens M, Vignes M: Low-frequency stimulation induces a new form of LTP, metabotropic glutamate (mGlu5) receptor- and PKAdependent, in the CA1 area of the rat hippocampus. Hippocampus 2006;16:345-360.

13 Ehrlich I, Humeau Y, Grenier F, Ciocchi S, Herry C, Lüthi A: Amygdala inhibitory circuits and the control of fear memory. Neuron 2009;62:757-771.

14 Quan M, Tian Y, Xu K, Zhang T, Yang Z: Post weaning social isolation influences spatial cognition, prefrontal cortical synaptic plasticity and hippocampal potassium ion channels in Wistar rats. Neuroscience 2010;169:214-222.

15 Palus M, Stefanovska A: Direction of coupling from phases of interacting oscillators: an information-theoretic approach. Phys Rev E Stat Nonlin Soft Matter Phys 2003;67: 055201.
16 Hlavácková-Schindler K, Palus M, Vejmelka M, Bhattacharya J: Causality detection based on information-theoretic approaches in time series analysis. Physics Reports 2007;441:146.

17 Palus M, Komárek V, Hrncír Z, Stěrbová K: Synchronization as adjustment of information rates: detection from bivariate time series. Phys Rev E Stat Nonlin Soft Matter Phys 2001;63:046211.

18 Palus M, Vejmelka M: Directionality of coupling from bivariate time series: how to avoid false causalities and missed connections. Phys Rev E Stat Nonlin Soft Matter Phys 2007;75:056211.

19 Vejmelka M, Palus M: Inferring the directionality of coupling with conditional mutual information. Phys Rev E Stat Nonlin Soft Matter Phys 2008;77:026214.

20 Li Y, Li X, Ouyang G, Guan X: Information flow among neural networks with Bayesian estimation. Chin Sci Bull 2007;52:2006-2011.

21 Musizza B, Stefanovska A, McClintock P, Palu M, Petrov i J, Ribari S, Bajrovi F: Interactions between cardiac, respiratory and EEG-delta oscillations in rats during anaesthesia. J Physiol 2007;580:315-326.

22 Palus M, Stefanovska A, Veber M: Causality between the amplitude and frequency of cardiac oscillations. Cardiovasc Engineer 2004 4:127-132. 
-23 Larson J, Lynch G: Induction of synaptic potentiation in hippocampus by patterned stimulation involves two events. Science 1986;232:985-988.

-24 Penttonen M, Buzsáki G: Natural logarithmic relationship between brain oscillators. Thalamus Relat Syst 2003;2:145-152.

25 Katz RJ: Animal model of depression: pharmacological sensitivity of a hedonic deficit. Pharmacol Biochem Behav 1982;16:965-968.

26 Willner P: Validity, reliability and utility of the chronic mild stress model of depression: a 10-year review and evaluation. Psychopharmacology (Berl) 1997;134:319-329.

27 Paxinos G, Watson C: The Rat Brain in Stereotaxic Coordinates. Amsterdam, Elsevier Academic Press, 2007.

28 Peng Z, Tse P, Chu F: A comparison study of improved Hilbert-Huang transform and wavelet transform: application to fault diagnosis for rolling bearing. Mech Syst Signal Process 2005;19:974-988.

29 Rosenblum M, Pikovsky A: Detecting direction of coupling in interacting oscillators. Phys Rev E Stat Nonlin Soft Matter Phys 2001;64:045202.

30 Rosenblum M, Cimponeriu L, Bezerianos A, Patzak A, Mrowka R: Identification of coupling direction: application to cardiorespiratory interaction. Phys Rev E Stat Nonlin Soft Matter Phys 2002;65:041909.
31 Barlow J: The Electroencephalogram: Its Patterns and Origins. Cambridge, MIT Press, 1993

32 Yokota Y: An approximate method for Bayesian entropy estimation for a discrete random variable. Conf Proc IEEE Eng Med Biol Soc 2005;1:99-102.

33 Nestler EJ, Hyman SE: Animal models of neuropsychiatric disorders. Nature Neurosci 2010;13:1161-1169.

34 Willner P: Chronic mild stress (CMS) revis ited: consistency and behavioural-neurobiological concordance in the effects of CMS. Neuropsychobiology 2005;52:90-110.

35 Jeong J, Gore J, Peterson B: Mutual informa tion analysis of the EEG in patients with Alzheimer's disease. Clin Neurophysiol 2001;112:827-835.

36 Steriade M: Neuronal Substrates of Sleep and Epilepsy. Cambridge, Cambridge University Press, 2003.

37 Welsh R, Chen A, Taylor S: Low-frequency bold fluctuations demonstrate altered thalamocortical connectivity in schizophrenia. Schizophr Bull 2008;36:713-722.

38 Goldman-Rakic P: Topography of cognition: parallel distributed networks in primate association cortex. Annu Rev Neurosci 1988; 11:137-156.
39 Zheng C, Quan M, Yang Z, Zhang T: Directionality index of neural information flow as a measure of synaptic plasticity in chronic unpredictable stress rats. Neurosci Lett 2011; 490:52-56

40 Brecht M, Sakmann B: Dynamic representation of whisker deflection by synaptic potentials in spiny stellate and pyramidal cells in the barrels and septa of layer 4 rat somatosensory cortex. J Physiol 2002;543:49-70.

41 Bruno RM, Simons DJ: Feedforward mechanisms of excitatory and inhibitory cortical receptive fields. J Neurosci 2002;22:1096610975.

42 Lewis D, Cruz D, Melchitzky D, Pierri J: Lamina-specific deficits in parvalbuminimmunoreactive varicosities in the prefrontal cortex of subjects with schizophrenia: Evidence for fewer projections from the thalamus. Am J Psychiatry 2001;158:14111422.

43 Gil Z, Connors BW, Amitai Y: Efficacy of thalamocortical and intracortical synaptic connections: quanta, innervation, and reliability. Neuron 1999;23:385-397.

44 Malenka RC, Bear MF: LTP and LTD: an embarrassment of riches. Neuron 2004;44:5-21.

45 Smith VA, Yu J, Smulders TV, Hartemink AJ, Jarvis ED: Computational inference of neural information flow networks. PLoS Comput Biol 2006;2:e161. 